\title{
LAPURDUTI
}

Euskal ikerketen aldizkaria | Revue d'études basques |

Revista de estudios vascos | Basque studies review

$12 \mid 2008$

Numéro XII

\section{C [texte intégral]}

C.A.D.E., C.B.L. Côte basque-loisirs, C.B.Ph., C.D.I.G.A., C.D.S. Bayonne, C.D.S. Boucau

\section{Q OpenEdition}

1 Journals

\section{Édition électronique}

URL : http://journals.openedition.org/lapurdum/1644

DOI : 10.4000/lapurdum. 1644

ISSN : 1965-0655

Éditeur

IKER

\section{Édition imprimée}

Date de publication : 1 février 2008

Pagination : 54-68

ISBN : 978-2-86781-617-8

ISSN : $1273-3830$

\section{Référence électronique}

«C [texte intégral] », Lapurdum [En ligne], 12 | 2008, mis en ligne le 24 mai 2010, consulté le 20 avril 2019. URL : http://journals.openedition.org/lapurdum/1644; DOI : 10.4000/lapurdum.1644

Ce document a été généré automatiquement le 20 avril 2019

Poitelon J.C. | IKER 


\section{C [texte intégral]}

C.A.D.E., C.B.L. Côte basque-loisirs, C.B.Ph., C.D.I.G.A., C.D.S. Bayonne, C.D.S. Boucau

1 C.A.D.E. Bulletin de liaison voir Collectif des associations de défense de l'environnement... Bulletin de liaison

2 C.B.L. Côte basque-loisirs. Le guide de vos loisirs. 21 déc. 1972/8 janv. 1973 (nº 1). Bayonne, $30 \times 11 \mathrm{~cm}$

En français

3 C.B.Ph. [puis C.B.Ph.N.]. Bulletin trimestriel, Côte basque philatélique [puis philatéliquenumismatique]. Irrégulier. [mai 1978] - août 1985 (n¹-20). janv. 1987 - nov. 1989 (n²1-29). - Biarritz, $30 \times 21 \mathrm{~cm}$ En français. - Publ. par: Côte basque philatélique [puis philatélique et numismatique] [Biarritz]. - Suite de : Côte basque philatélique. Bulletin de la société

4 C.D.I.G.A. Bulletin de liaison. déc. 1996 - janv. 2000 ( $\left.n^{\circ} 1-4\right)$. - Anglet, $30 \times 21 \mathrm{~cm}$ En français. - Publ. par: Centre de documentation informatique de généalogie d'Anglet [Anglet]

5 C.D.S. Bayonne. Centre des démocrates sociaux. Section de Bayonne, bulletin d'information [puis Bulletin d'information de la Section Bayonne, Boucau, Saint-Pierred'Irube]. Trimestriel. févr. 1983 - août 1986 ([1 $\left.1^{\mathrm{re}}\right]-4^{\mathrm{e}}$ année, $\left.\mathrm{n}^{\circ} 1-13\right)$. mars 1988 (5 ${ }^{\mathrm{e}}$ année, $\mathrm{n}$ $\left.{ }^{\circ} 14\right)$. - Bayonne, $30 \times 21 \mathrm{~cm}$

En français. - En 1988 reparaît à l'occasion de l'élection présidentielle d'avril pour soutenir la candidature de Raymond Barre

6 C.D.S. Boucau. Bulletin. Bimestriel. sept. 1984 - janv. 1986 (n¹-8). - Boucau, $30 \times 21 \mathrm{~cm}$ En français. - Publ. par: Centre des démocrates sociaux. Section de Boucau [Boucau]. Remplacé par : Boucau

7 C.E.M.J.-infos. La Commission extra-municipale jeunesse. juin 2001 ( $\left.n^{\circ} 0\right)$. - Bayonne, $30 \times 21 \mathrm{~cm}$ En français 
8 Cagouille (La) en Pays basque. Revue bi-mestrielle de l'Amicale des Charentais en Pays basque. mai 1961 - mars 1962 (n¹-6) [?]. - Biarritz, $27 \times 21 \mathrm{~cm}$ En français; quelques textes en dialectes des Charentes

9 Cahier (Le) d'Ikas-bi. Irrégulier. mai $1997\left(n^{\circ} 16\right) \rightarrow$. Saint-Jean-de-Luz, $30 \times 21 \mathrm{~cm}$ En français; quelques textes basques. - Suite de : Ikas-bi... Cahier

10 Cahier (Le) de Biarritz-culture. Bimestriel. mars-juil. 1992 (3 nº). sept. 1992 - juil./ août 1997 (n॰[1]-26). - Biarritz, $30 \times 23$ puis $28 \times 20 \mathrm{~cm}$ Jusqu'en juil. 1992 a paru sous le titre: Arts, spectacles, \{loisirs\}. Le cahier de Biarritz-culture. - En français. - Contenu aussi dans : Biarritz-magazine. - Devient : Kulturaldia

11 Cahier (Le) de Biarritz-culture. Irrégulier. nov./déc. $2001\left(\mathrm{n}^{\circ} 50\right) \rightarrow$. Biarritz, $21 \times 15 \mathrm{~cm}$ Avec un n'spécial annuel présentant la saison culturelle. - Titre basque: Kulturaldia. - En français; quelques textes basques. - Suite de: Kulturaldia

12 Cahiers (Les) d'Ikas-bi. Pour le bilinguisme dans l'enseignement public en Pays basque. Trimestriel de l'association Ikas-bi et de Parents d'élèves Pays basque. avr. 1989 - déc. 1991 (n¹-5). - Saint-Jean-de-Luz, $30 \times 21 \mathrm{~cm}$

En français; quelques textes basques. - Devient : Ikas-bi... Cahier

13 Cahiers (Les) d'information de l'U.M.P. Côte basque, VIe circonscription des Pyrénées-Atlantiques. déc. 2003/janv. 2004 $\rightarrow$. Biarritz, $30 \times 21 \mathrm{~cm}$

En français. - Publ. par: Union pour un mouvement populaire. $6^{\mathrm{e}}$ circonscription des Pyrénées-Atlantiques [Biarritz]

14 Cahiers (Les) de l'Académie du chocolat. Semestriel puis irrégulier. 15 mai $1999\left(n^{\circ} 1\right) \rightarrow$. Bayonne, $30 \times 21 \mathrm{~cm}$

En français

15 Cahiers (Les) de l'Adour. mars 1986 - févr. 1989 (n¹-5). - Bayonne, $21 \times 15 \mathrm{~cm}$ Avec $2 n^{\circ}$ spéciaux intitulés: Plume d'argent 1985 et Plume d'argent 1986. - En français. Devient : Nouveaux (Les) cahiers de l'Adour

16 Callejon. Annuel, photos et bilan..., Sud-Ouest. 1980 - 1986 ( $\left.n^{\circ} 1-7\right)$. - Orthez, Biarritz, $22 \times 17 \mathrm{~cm}$

En français. - Photographies de Jacques Cathalaa et Bernard Hiribarren; textes de Claude Pelletier. - Les années 1987 et 1988 auraient paru également, sous un titre sans doute différent

17 Cambo après la pluie... «Le journal pensant bien». juin 1994 - janv. $1995\left(1^{\text {re }}-2^{\mathrm{e}}\right.$ année, $\mathrm{n}^{\circ}$ 1-3). mars 2001 (2e année, $\left.{ }^{\circ} 4\right)$. - Cambo-les-Bains, $30 \times 21 \mathrm{~cm}$ En français. - En 2001 reparaît à l'occasion des élections municipales de mars

18 Cambo-avenir. R.P.R. - P.R. févr. - mars 1983 (n¹-2). - Cambo-les-Bains, $35 \times 25 \mathrm{~cm}$ En français. - Publ. à l'occasion des élections municipales de mars 1983 pour soutenir la liste «Cambo-avenir»

19 Cambo demain. Au service de tous. Denen zerbitzari. [mars 1989] ( $\left.{ }^{\circ} 1\right)$. - Cambo-lesBains, $43 \times 30 \mathrm{~cm}$

En français; quelques textes basques. - Publ. à l'occasion des élections municipales de mars 1989 pour soutenir la liste «Cambo demain»

20 Cambo en expansion. Bulletin officiel municipal. mars $1965\left(n^{\circ} 1\right)-[. .$.$] . - Cambo-les-$ Bains, Paris, $27 \times 19 \mathrm{~cm}$

En français. - En 1981 devient : Cambo-les-Bains. Bulletin municipal 
21 Cambo-infos. Lettre d'informations municipales de la commune de Cambo-les-Bains. Irrégulier. nov. 1995 - janv./févr. 2001 (n¹-9). - Cambo-les-Bains, 30 × $21 \mathrm{~cm}$ En français. - Suite de : Cambo-les-Bains. Bulletin municipal Cambo-les-Bains. Bulletin municipal. Irrégulier. mai 1981 - juil. 1988 (n¹-3). $1^{\mathrm{er}}$ sem. 1990. $1^{\mathrm{er}}$ sem. 1991. - Cambo-les-Bains, $30 \times 21 \mathrm{~cm}$ En français. - Suite de : Cambo en expansion. - En 1995 devient : Cambo-infos Cambo-revivre. Bulletin du Comité inter-sanas de la Fédération nationale de lutte antituberculeuse (F.N.L.A.) puis Bulletin du comité local de la F.N.L.A. puis Bulletin du Comité Cambo-Côte basque de l'A.M.I. (Association nationale de défense des malades, invalides et infirmes). Irrégulier. [...]. - [Cambo-les-Bains], de $31 \times 21$ à $26 \times 18 \mathrm{~cm}$ Paraissait déjà en juil. 1957 et encore en 1972. - En français

Camuscope. printemps 1997 - 1998 (n¹-3). - Bayonne, $30 \times 21 \mathrm{~cm}$

En français. - Publ. par : Collège Albert Camus [Bayonne]. - Suite de : Cocktail

Canard (Le) local. \{Mensuel de l'information culturelle au Pays basque\}. 18 oct./15 nov. 1993 - [juin/sept. 1994] (n¹-9). - Bayonne, $21 \times 15$ puis $27 \times 15 \mathrm{~cm}$

En français. - En 1997 éléments repris par : đEil (L') sauvage

Cancans et autres potins d'Abbadia. Irrégulier. [sept. $\left.1998\left(\mathrm{n}^{\circ} 1\right)\right] \rightarrow$. Hendaye, $30 \times 21$ puis $21 \times 15 \mathrm{~cm}$

Les 4 premiers $n^{\circ}$ ont paru sous le titre: Cancans d'été..., Cancans d'automne..., Cancans d'hiver..., Cancans de printemps...; à partir d'août 2003 titre basque : Abbadiako kutzukeriak. - En français; à partir d'oct. 2001 quelques textes basques. - Publ. par : Amis (Les) d'Abbadia; en basque : Abbadiako adixkideak

27 Cantau-actualités. Mensuel ou bimestriel. avr. 1968 - mai/juin 1971 (n¹-19) [?]. - [Anglet], $28 \times 22 \mathrm{~cm}$

En français. - Publ. par : Lycée professionnel Cantau [Anglet]

Canton (Le) d'Anglet-Nord. Annuaire cantonal. 1992/93. - [Anglet], Paris, $21 \times 14 \mathrm{~cm}$

La couv. porte en tête : Département des Pyrénées-Atlantiques. - En français

Canton (Le) d'Anglet-Sud. Annuaire [puis Agenda] cantonal. 1990 - 1991. - [Anglet], Paris puis Anglet, $21 \times 14 \mathrm{~cm}$

En 1991 titre de couv.: Anglet-Sud. Agenda cantonal. - La couv. porte en tête en 1990: Département des Pyrénées-Atlantiques, et en 1991 : Conseil général. - En français

30 Cap Sud. Actuel Pays basque magazine. Mensuel d'actualité économique et touristique franco-espagnol. déc. 1973 - déc. 1974 ( $\left.n^{\circ} 10-22\right)$. - Biarritz, $27 \times 21$ puis $30 \times 21 \mathrm{~cm}$

En français; quelques textes espagnols. - Suite de : Actuel Pays basque magazine

31 Carlton (Le). [puis Art et culture en Côte basque]. juin 1993 - 1994 (nº1-3). - Biarritz, $44 \times 32 \mathrm{~cm}$

En français. - A partir du $n^{\circ} 2$ publ. par : Association Patrimoine Côte basque [Biarritz]

32 Carnet de bord de la solidarité. 1984 [?] - 1986 (n¹-3). - Bayonne, $30 \times 21 \mathrm{~cm}$ En français. - Publ. par: Secours populaire français. Fédération Côte basque - Sud des Landes [Bayonne]

33 * Cassindex. [déc. 1986] - [...]. - [Bayonne], $30 \times 21 \mathrm{~cm}$ En français. - Rédigé par des élèves du Lycée René Cassin [Bayonne]

Catéchistes ensemble. [puis Bulletin de liaison des catéchistes du Pays basque et des pays de l'Adour]. $3 n^{\circ}$ par an puis trimestriel. [déc. 1977] $\left(n^{\circ} 1\right) \rightarrow$. Bayonne, $30 \times 21 \mathrm{~cm}$ 
En français; à partir de déc. 1990 quelques textes basques. - Publ. par: Centre diocésain de catéchèse [Bayonne]

Ce mois-ci. Mensuel ou irrégulier. mars 1991 (n¹) - 1993. oct. 1996 - sept. 1998 (n¹7-35). Bayonne puis Hasparren, $30 \times 21$ puis $21 \times 15 \mathrm{~cm}$

En français. - A partir de 1996 publ. par: Radio bonne humeur [Hasparren]. - Voir aussi Igandea

* Cegecoba-infos. [puis Cegecoba. Le journal]. Le journal d'informations du Cegecoba [puis Le développement des artisans et commerçants]. Irrégulier. juil. 1999 - oct. 2002 (vol. 1-22). 2003 (vol. 1) $\rightarrow$. Anglet, $30 \times 21 \mathrm{~cm}$

En français. - Publ. par : Centre de gestion agréé de la Côte basque [Anglet]

37 Centre culturel et social de Boucau et Tarnos. Le bulletin de l'histoire locale. déc. 1996 - avr. $2002\left(n^{\circ} 1-2\right) .-$ [Boucau], $30 \times 21 \mathrm{~cm}$

En français

Centre d'animation culturelle de la Côte basque. Propos voir Propos

Centre de documentation informatique de généalogie d'Anglet... voir C.D.I.G.A.

Centre de gestion agréé de la Côte basque... voir Cegecoba...

Centre des démocrates sociaux... voir C.D.S...

Centre du volontariat Pays basque... voir Gazette (La) du bénévole et Gazette (La) du Centre du volontariat...

3 Cercle aquariophile du Bas-Adour. Bulletin de liaison. Irrégulier. 2 avr. 1980 - janv. 1983 ( $\left.\mathrm{n}^{\circ} 1-9\right) .1983\left(2 \mathrm{n}^{\circ}\right)$ [?]. - Bayonne puis Biarritz, $30 \times 21 \mathrm{~cm}$

En français. - Reprend certains éléments de: Cercle aquariophile des Pyrénées. Bulletin mensuel [Lescar]

$44 *$ Cercle d'études et informations d'Urcuit. Irrégulier. 25 janv. $1982\left(\mathrm{n}^{\circ} 1\right)$ - [...]. janv. $1991\left(n^{\circ} 14\right)$ - [...]. - Urcuit, $30 \times 21$ puis $21 \times 15 \mathrm{~cm}$

Bulletin politique local. - En français; quelques textes basques

Cercle généalogique du Pays basque et Bas-Adour. Labourd, Basse-Navarre, Soule. Pays du Bas-Adour: Orx et Orthe [puis Orthe, Gosse et Seignanx]. Bulletin \{de liaison\} [puis Bulletin trimestriel]. Annuel puis semestriel puis trimestriel. 1986 - mars 2002 ( $\left.\mathrm{n}^{\circ} 1-35\right)$. Bayonne, $30 \times 21 \mathrm{~cm}$

Avec un $n^{\circ}$ spécial de mars 2002 intitulé: Construction de la Villa Eugénie, Biarritz, 1854 1861, par André Lebourleux. - En français. - En [avr. 2000] (n²8) a paru sous une couv. commune avec le $n^{\circ}$ de même date de : Jakintza. - Devient : Généalogie et histoire des familles. Pays basque-Adour maritime

Cette semaine chez nous. Flash info hebdomadaire de Biarritz-tourisme. Hebdomadaire puis irrégulier. $12 / 19$ oct. 1998 - 12/18 nov. 2001 (n¹-130). 26 nov./2 déc. 2001 - 14/20 juin 2004. - [Biarritz], $30 \times 21 \mathrm{~cm}$

En français

Cèu (Lo) au Paìs basco voir Ciel (Le) au Pays basque

Chambre de commerce et d'industrie de Bayonne. Feuilles d'informations techniques voir Feuilles d'informations techniques

Chambre de commerce et d'industrie de Bayonne Pays basque. Rapport d'activité[s]. Annuel. $1995 \rightarrow$. Bayonne, $30 \times 21 \mathrm{~cm}$ 
Certaines années ont paru sous le titre: Rapport d'activité[s]. Chambre de commerce... - En français. - De 1995 à 2002 constitue les nº40, 448, 452, 454, 455 et 457 à 459 de: Activités en Pays basque

51 Chambre F.N.A.I.M. de l'immobilier du Pays basque. Info Pays basque voir Info Pays basque

Chambre F.N.A.I.M. des professionnels immobiliers des Pyrénées-Atlantiques-Côte basque. Info Côte basque voir Info Côte basque

Champignon. Auto, moto, kart, sport et loisir. Le mensuel de tout ce qui roule vite dans notre région. juil. $1984\left(\mathrm{n}^{\circ} 1\right)$ [?]. - Mouguerre, $32 \times 23 \mathrm{~cm}$

En français

54 Charivari (Le) des Pyrénées. Hebdomadaire. 15/22 mars - 19/25 juil. 1951 ( $\mathrm{n}^{\circ} 1-19$ ). 22 mai, 9 oct. 1952 (n²0, 21). 9 janv., 9 avr. 1953 (n²2, 23). - Bayonne, $44 \times 30 \mathrm{~cm}$ En français

55 Château de Brindos. été 1997 - printemps 1998 (n¹-2). - Anglet, $42 \times 30 \mathrm{~cm}$ En français. - Publ. par l'hôtel : Château de Brindos [Anglet]

$56{ }^{*}$ Check le B.A.B. Magazine alternatif. Irrégulier. été $2004\left(n^{\circ} 1\right) \rightarrow$. Anglet puis Bayonne, $21 \times 15 \mathrm{~cm}$

En français. - Publ. par : Check le B.A.B. [Anglet]

57 Chêne (Le) de la Côte basque. Bulletin d'information [puis Mensuel d'information] du Mouvement national républicain. Mensuel. oct. 2000 - avr./juin 2003 (n¹-21). - Anglet, $30 \times 21 \mathrm{~cm}$

En français. - Publ. par : Mouvement national républicain. Fédération Côte basque [Anglet]

Chéraute. Bulletin d'informations municipales. juil. 1993. - Chéraute, $30 \times 21 \mathrm{~cm}$

En français. - En 2003 devient : Chéraute - Sohüta. Bulletin municipal

* Chéraute-Sohüta. Bulletin municipal. Semestriel. mars/juin $2003\left(n^{\circ} 1\right) \rightarrow$. Chéraute, $30 \times 21 \mathrm{~cm}$

En français. - Suite de : Chéraute. Bulletin d'informations municipales

Chiberta voir Tee (Le) du lac

$61{ }^{*}$ Chiche. Le journal qui a du poids... [...]. - Saint-Jean-de-Luz, $30 \times 21 \mathrm{~cm}$

Paraissait en 1988/89 (n4-5). - En français. - Rédigé par des jeunes de l'Aumônerie du Lycée Maurice Ravel [Saint-Jean-de-Luz]

Chien velu. Revue [puis Revue au poil] pour les enfants de 8 à 14 ans [puis à 14 ans et demi]. Trimestriel. $1^{\text {er }}$ trim. 1997 - juin 1999 (n¹-10). - Anglet, $21 \times 15 \mathrm{~cm}$

Avec un nspécial de déc. 1998. - En français. - Publ. par : Lire c'est vivre [Anglet]. - Voir aussi Train de nuit

63 Chikitoys. Bulletin de liaison trimestriel... [févr.] - avr. 1961 (n¹-2) [?]. - Bayonne, $32 \times 25 \mathrm{~cm}$

En français

$64{ }^{*}$ Chimère. Revue d'information du $1^{\text {er }}$ R.P.I.M.A. Mensuel ou irrégulier. [...]. - [Bayonne], $30 \times 21 \mathrm{~cm}$

Paraissait en 1997. - En français. - Publ. par : $1^{\text {er }}$ Régiment de parachutistes d'infanterie de marine [Bayonne]

Chlorophil [ou Chlorophyl]. Irrégulier ou annuel. déc. $1989\left(\mathrm{n}^{\circ} 1\right) \rightarrow$. Boucau puis Boucau, Tarnos, $21 \times 15$ puis $30 \times 21 \mathrm{~cm}$ 
En français. - Publ. par: Information-défense de l'environnement de l'Adour et du littoral (I.D.E.A.L.) [Boucau puis Boucau, Tarnos]

Christien (Le). Informations, actions, études... bi-mensuel de Foi-Espérance-Agapé [puis Mensuel de l'Ordre christien puis Bi-mestriel de l'Ordre christien]. 2 janv. 1974 - nov./déc. 1991 (n¹-227). - Souraïde, $30 \times 21 \mathrm{~cm}$

En français. - Publ. par : Compagnie chevaleresque de l'Ordre christien [Souraïde]

Ch'timi (Le). Bulletin de [puis Edité par puis Périodique édité par] l'Association des amis du Nord et du Pas-de-Calais de la Côte basque [ou de la Côte basque et du Béarn ou de la Côte basque et du Sud des Landes]. De 2 à 4 fois par an. [avr./mai 1954] ( $\left.n^{\circ} 1\right) \rightarrow$. Biarritz ou Bayonne ou Anglet, $24 \times 16$ puis $27 \times 21$ puis $30 \times 21 \mathrm{~cm}$

En français; quelques textes en dialectes du Nord de la France. - Publ. par : Amis (Les) du Nord et du Pas-de-Calais de la Côte basque [puis de la Côte basque et du Sud des Landes] [Biarritz ou Bayonne ou Anglet]. - Reprend certains éléments de: Ch'timi (Le) [Pau]. - De mai 1971 à janv. 1974 contient : Ch'timi (Le) en Béarn

Ch'timi (Le) en Béarn. 3 fois par an. [mai 1971] - janv. 1974 (n[1] - 9A). - Biarritz, $27 \times 21 \mathrm{~cm}$

En français. - Reprend certains éléments de: Ch'timi (Le). Bulletin de l'association «Les Amis du Nord et du Pas-de-Calais» résidant dans les Pyrénées-Atlantiques, paru à Pau de 1947 [?] à 1962. - Contenu dans : Ch'timi (Le) [Biarritz]

69 Ciboure. Bulletin municipal [puis Bulletin d'information de la mairie]. Irrégulier. janv. 1990 - août 1992 (n¹-3). sept. 1993. août 1994. juin 1996 - nov. 2000. - Ciboure, $30 \times 21 \mathrm{~cm}$ En sept. 1993 et août 1994 a paru sous le titre : Bulletin (Le) de Ciboure. - En français. - Suite de : Bulletin municipal. Ciboure. - Devient : Ziburu-Ciboure. Bulletin d'information...

Ciboure demain. déc. 1988 - mars 1989. - Ciboure, 30 × $21 \mathrm{~cm}$

En français. - Publ. à l'occasion des élections municipales de mars 1989 pour soutenir la liste «Ciboure demain». - Reprend certains éléments du bulletin de même titre publ. en 1985 et 1986; voir la notice suivante

Ciboure demain. Organe d'information de l'opposition libérale. juin $1985\left(n^{\circ} 1\right)$ - 1986 [?]. - Ciboure, $30 \times 21 \mathrm{~cm}$

En français. - Publ. par : Ciboure demain [Ciboure]. - Certains éléments repris en 1988 par un bulletin de même titre; voir la notice précédente

Ciel (Le) au Pays basque. Ephémérides. Bimestriel. juil./août $1996\left[\mathrm{n}^{\circ} 1\right] \rightarrow$. Anglet puis Biarritz, $21 \times 15 \mathrm{~cm}$

A partir de mars/avr. 1999 titre basque : Zerua Euskal herritik ikusirik; à partir de 2002 titre gascon : Cèu (Lo) au Paìs basco. - En français. - Publ. par : Société d'astronomie populaire de la Côte basque puis Astronomie - Côte basque [Biarritz]

73 Cigale des pins. 1956/57 - 1958/59 [?]. - Anglet, $28 \times 21 / 23 \mathrm{~cm}$

En français. - Publ. par : Pensionnat Stella Maris [Anglet]

Ciné-club de Biarritz. Programme \{du mois de\} [ou Mois de]... Mensuel. oct. [1958] [nov. 1961]. déc. 1961 (18e année, n³) - mars 1976 [?]. - Biarritz, $27 \times 21$ puis $30 \times 21 \mathrm{~cm}$ En français. - Suite de : Bulletin du Ciné-club de Biarritz

Ciné-infos [puis Ciné-infos-spectacles puis Ciné-spectacles-infos]. Mensuel. mars 1989 ( $\left.{ }^{\circ} 1\right)$

$\rightarrow$. Bayonne, $21 / 23 \times 15$ puis $30 \times 21 \mathrm{~cm}$

En français. - Publ. par : Entr'actes [Bayonne] 


\section{$\rightarrow$. Anglet, $30 \times 21 \mathrm{~cm}$}

Partie du titre en gros caractères: Vivre ensemble. - En français. - Publ. par : Citoyens en Adour-Pyrénées - Vivre ensemble [Anglet]

* Clandestin (Le). Journal lycéen édité par le groupe anarchiste Durruti, G.A.D. [puis Journal de jeunes...]. [janv./mars - mai 1983] (n¹-2) [?]. - Bayonne, $30 \times 21 \mathrm{~cm}$ En français. - Suppl. de : Emancipations

$21 \times 21$ puis $30 \times 21 \mathrm{~cm}$

En français. - Publ. par : Zabalki [Hasparren]

Programme. Mensuel. 22 déc. 1993/25 janv. 1994 [n¹] $\rightarrow$. Saint-Jean-Pied-de-Port $30 \times 21 \mathrm{~cm}$

En français ou en basque et français. - Publ. par : Garazikus [Saint-Jean-Pied-de-Port]

Paraissait déjà en 1995. - En français. - Publ. par : Argitze [Saint-Palais]

1962 - mai 1963 (n¹-6). nov. 1963 - févr. 1964 (n7-10). [sept. 1964 - mars 1967] (11 nº). Hendaye, $22 \times 14$ puis $25 \times 18 \mathrm{~cm}$

En oct. et nov. 1962 a paru sous le titre : 4 [Quatre] (Les) clochers de la Bidassoa. - A partir de janv. 1963 titre de couv. : 5 [Cinq] (Les) clochers sur la Bidassoa. Hendaye-Plage, Paroisse de $\mathrm{S}^{\text {te }}$ Anne, Biriatou, Urrugne, Hendaye-Ville, Paroisse de $\mathrm{S}^{\mathrm{t}}$ Vincent, Béhobie. - En basque et français. - Pour Hendaye remplace : Clocher (Le) hendayais. - A partir de sept. 1964 texte en partie identique à celui de : Denak argian qui l'absorde en mai/juin 1967

Clé (La) du logis. Offre de locations entre particuliers. Hebdomadaire. 3 août 1993 - 1995. Bayonne, $30 \times 21 \mathrm{~cm}$

En français. - Remplace : Offres (Les) des propriétaires Hestia [1989 - 1993]

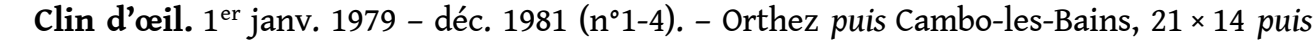
$29 \times 21 \mathrm{~cm}$

Recueils de textes et de dessins d'élèves des collèges d'Orthez puis de Cambo, réalisés à l'initiative de leur professeur d'anglais et impr. par l'Imprimerie spéciale de La Ronde. - En anglais; quelques textes français et espagnols

Clin d'œil. Le journal d'information des jeunes. déc. $2000\left(\mathrm{n}^{\circ} 0\right)$. - Saint-Etienne-deBaïgorry, $30 \times 21 \mathrm{~cm}$

En français. - Publ. par : Comité Izpegi [Saint-Etienne-de-Baïgorry]

Clin d'œil. Lettre d'informations de la mutuelle P.A.M. et de la Mutrans... Mensuel. 14 mars 1997 - 15 sept. 2000 ( $\left.{ }^{\circ} 1-43\right)$. - Bayonne, $30 \times 21 \mathrm{~cm}$ Avec 6 suppl. publ. de mai 1997 à août 1999. - En français. - Publ. par : Pyrénées-Atlantiquesmutualité (P.A.M.) [Bayonne] et Mutrans [Mutuelle des travailleurs non salariés] [Bayonne]. - Voir aussi Dyna-P.A.M. 
Clocher (Le) hendayais. Mensuel. nov. 1949 - juin 1962 ( $\left.n^{\circ} 1-118\right)$. - Hendaye, $22 \times 14 \mathrm{~cm}$ Bulletin paroissial de Saint-Vincent [Hendaye]. - En français. - Remplacé par : 5 [Cinq] (Les) clochers de la Bidassoa

Club Boucau 95. Irrégulier. oct. 1989 - déc. 1994 ( $1^{\mathrm{re}}-5^{\mathrm{e}}$ année, $\left.\mathrm{n}^{\circ} 1-12\right)$ - Boucau, $30 \times 21 \mathrm{~cm}$

En français. - Publ. par: Club Boucau 95 [Boucau]. - Remplace: Boucau. - Remplacé par : Boucau 2001

Club de la presse, Pays basque, Euskal herria, País vasco. [Annuaire]. Annuel. $1992 \rightarrow$. Bayonne, $21 \times 11 \mathrm{~cm}$

En basque, espagnol et français. - Publ. par : Club de la presse du Pays basque; en basque: Euskal herriko prentsa elkartea; en espagnol : Club de la prensa del País vasco [Bayonne]

Club-foyer de l'age d'or. Bulletin d'informations. Mensuel puis irrégulier. oct. 1987 ( $\left.n^{\circ} 1\right)$ - mars $1991\left(\mathrm{n}^{\circ} 2\right)$ [?]. - Biarritz, $30 \times 21 \mathrm{~cm}$

Jusqu'en févr. 1988 a paru sous le titre : Foyer-club de l'age d'or. Bulletin d'information. - En français. - Devient : Clubs de loisirs de Biarritz. Bulletin d'informations

Club gramontois, Bidache. Bulletin d'information. Mensuel ou irrégulier. oct. 1995 ( $\mathrm{n}^{\circ} 1$ )

$\rightarrow$. Bidache, $21 \times 15$ ou $30 \times 21 \mathrm{~cm}$

En français

Club Léo Lagrange \{de Bayonne. Bulletin\}. Irrégulier. 1968 ( $\left.n^{\circ} 1\right)$ - [...]. mai - déc. 1980 (n ${ }^{\circ} 11-12$ ). - Bayonne, $27 \times 21$ puis $30 \times 21$ puis $15 \times 21 \mathrm{~cm}$ En français. - Devient : Shuss

Clubs de loisirs de Biarritz. Bulletin d'informations. Trimestriel. oct./nov. 1991 - avr./ juin $1992\left(n^{\circ} 1-3\right)$. - Biarritz, $30 \times 21 \mathrm{~cm}$

En français. - Publ. par : Comité de l'age d'or [Biarritz]. - Suite de: Club-foyer de l'age d'or. Bulletin d'informations. - Devient : Petit (Le) journal [Biarritz]

Cocktail. Le journal du Collège Albert Camus. Irrégulier. mars 1993 (n¹) - 1995/96. Bayonne, $30 \times 21 \mathrm{~cm}$

En français. - Reprend les éléments de : Echos liés. - Devient : Camuscope

Coin (Un) de Colombie. Bulletin d'information. Irrégulier. août $1993\left(n^{\circ} 1\right) \rightarrow$. Bayonne, Biarritz puis Bayonne, $30 \times 21 \mathrm{~cm}$

En français. - Publ. par : Coin (Un) de Colombie [Bayonne]

Collectif Combo. 1999 - 2001 ( $\left.n^{\circ} 1-3\right)$. - Anglet, $15 \times 21$ puis $21 \times 15 \mathrm{~cm}$

En français. - Publ. par : Collectif Combo [Anglet]. - Devient : Redux. - Voir aussi Guide du skater

Collectif des associations de défense de l'environnement Pays basque-Sud des Landes. Bulletin de liaison. Mensuel ou irrégulier. [mai 1992] $\left(\mathrm{n}^{\circ} 1\right) \rightarrow$. Bayonne, $30 \times 21 \mathrm{~cm}$ Avec un $n^{\circ} 0$ de [mai 1992]. - Le $n^{\circ} 21$ a paru sous le titre: C.A.D.E. Bulletin de liaison. - En français

99 Comité d'établissement Dassault-Bréguet. Commission culturelle. Usine de Biarritz. Bulletin d'information. Irrégulier. févr. $1979\left(\mathrm{n}^{\circ} 1\right)$ - 1987 [?]. - Biarritz, $30 \times 21 \mathrm{~cm}$ En français. - Voir aussi Bulletin d'information sur la formation

Comité de l'age d'or. Le Petit journal voir Petit (Le) journal

Comité pour la défense des droits de l'homme en Pays basque. Bulletin [puis Bulletin trimestriel]. Irrégulier puis trimestriel. avr. 1986 - juin 1992 ( $\left.n^{\circ}[1]-23\right)$. - Bayonne, 
$30 \times 21 \mathrm{~cm}$

A partir de juin 1991 porte en tête : Euskal herriko giza eskubideen babes elkartea. - En français. - Devient : Jakilea

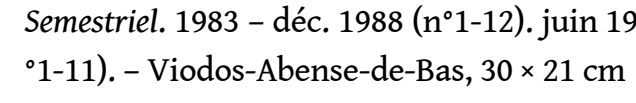

En français. - Devient : Viodos-Abense. Notre commune

* Communistes (Les) du canton d'Ustaritz. Irrégulier. 4 oct. 2001 (n¹). [déc. 2002] $\rightarrow$. [Ustaritz], $30 \times 21 \mathrm{~cm}$

En oct. 2001 a paru sous le titre : Nouvelles (Les) du Pays basque. Bulletin d'information de la Section du Parti communiste français de Cambo-Ustaritz. - En français

Confetti-Côte basque-magazine. Bimensuel de culture et de loisirs [puis De Bayonne à St-Sébastien, Pau et Dax [puis Pau, Dax, Capbreton, Hossegor]...]. Bimensuel. 2/15 juin 1993 $\left(n^{\circ} 1\right)$ - 1994. - Biarritz, $21 \times 15 \mathrm{~cm}$ En français

Conjoncture économique Pays basque. Une publication trimestrielle de la Chambre de commerce et d'industrie de Bayonne Pays basque. mars 1998 - mars 2002 (n¹-16). Bayonne, $30 \times 21 \mathrm{~cm}$ En français 
119 Contact. Herriko berriak. Espelette, bulletin d'informations municipales. Semestriel. [juil. 1983 - janv. 1986] (n $1-6)$. - Espelette, $30 \times 21 \mathrm{~cm}$

En français. - Parait parallèlement à : Biper berri. - En 1989 devient : Ezpeleta

Contact. Journal des participants des spectacles de Bidache. Mensuel puis trimestriel. janv. 1990 - juil. 1991 ( $\left.n^{\circ} 1-10\right)$. - Bidache, $30 \times 21 \mathrm{~cm}$

En français. - Publ. par: Spectacles (Les) du château de Bidache [Bidache]. - Remplace: Echos (Les) des coulisses...

121 Contact santé. Bulletin d'information de la Caisse primaire d'assurance maladie de Bayonne. Trimestriel. été $2002\left(\mathrm{n}^{\circ} 1\right) \rightarrow$. Bayonne, $30 \times 21 \mathrm{~cm}$

En français

122 Corde magno. \{Lettre aux oblats et amis de l'Abbaye N.-D. de Belloc\}. Trimestriel. 3 mai 1954 - nov. 1988 (n $1-131)$. - Urt, $31 \times 21$ puis $21 \times 16 \mathrm{~cm}$

En français. - Pour l'éd. basque voir Othoizlari. - Voir aussi Abbaye N.-D. de Belloc. Lettre aux oblats. - Devient : Voix de Belloc

Côte basque. Le magazine des vacances voir Côte basque-Landes-magazine

Côte basque accueil voir Accueils (Les) de la Côte basque

Côte basque démocratique. Mensuel des sections de la Côte du Parti communiste français. févr. 1976 - oct. 1984 ( ${ }^{\circ} 1$-94) [?]. - Bayonne, $32 \times 27$ puis $43 \times 30 / 32$ puis $30 \times 21 \mathrm{~cm}$

En français. - Fondé à l'occasion des élections cantonales de mars 1976

Côte basque-été voir Pays basque-été

Côte basque-Landes-magazine. \{Le magazine des vacances\}. Trimestriel. Pâques 1989 [1990] (nºspécial «fêtes de fin d'année»). - Biarritz, $30 \times 21 \mathrm{~cm}$

Le n ${ }^{\circ}$ de Pâques 1989 a paru sous le titre : Côte basque. Le magazine des vacances. - En français. - Suite de : Gazette (La) de la Côte basque

Côte basque-loisirs [1972] voir C.B.L.

Côte basque-loisirs. Magazine hebdomadaire des loisirs, spectacles [puis des loisirs, spectacles, télévisions] et bonnes adresses [puis Magazine hebdo des loisirs, gratuit]. Hebdomadaire ou irrégulier. 13 juil. $1983-26 \mathrm{mai} / 1^{\mathrm{er}}$ juin 1988 ( $\left.\mathrm{n}^{\circ} 1-199\right)$. - Bayonne puis Biarritz puis Anglet, $22 \times 14 / 16$ puis $22 \times 10$ puis $21 \times 14 \mathrm{~cm}$ En français

130 Côte basque-madame. Trimestriel. déc. 2003/févr. 2004 (n¹) [?]. - Bordeaux, $29 \times 21 \mathrm{~cm}$ En français

131 Côte basque-magazine. Mensuel. juil./août $2002\left(\mathrm{n}^{\circ} 1\right) \rightarrow$. Anglet, $21 \times 15$ puis $24 \times 17 \mathrm{~cm}$ En français

132 * Côte basque-magazine. Actualités basco-béarnaises [puis Supplément de «La Voix du Pays Basque»]. 1966 - 1973 [?]. - Anglet puis Biarritz, Bayonne, $27 \times 21 \mathrm{~cm}$ En français. - Dir. publ. Georges de Styn. - Devient : Atlantique-Pyrénées Côte basque philatélique. Bulletin de la société. [...] - 1977. - Biarritz, $30 \times 21 \mathrm{~cm}$ Paraissait déjà en mars 1967 (n²1). - En français. - Devient : C.B.Ph.

134 Côte basque-rassemblement. [puis Bulletin de liaison des sections R.P.R. de la VI ${ }^{\mathrm{e}}$ circonscription des Pyrénées-Atlantiques]. Irrégulier. févr. 1992 - nov. 1994 (n¹-17). Biarritz, $30 \times 21 \mathrm{~cm}$ Avec un no de janv. 1992. - Certains nºnt paru sous le titre: Lettre (La) de Côte basque- 
rassemblement. - En français. - Publ. par: Rassemblement pour la République. $6^{\mathrm{e}}$ circonscription des Pyrénées-Atlantiques [Biarritz]. - Reprend certains éléments de: Echos de Biarritz. - En 1998 certains éléments repris par : Echos (Les) de Biarritz et du Pays basque

Côte basque-soir. Résistance républicaine [puis Quotidien d'information. Résistance républicaine]. Quotidien. 31 juil. $1948\left(5^{\mathrm{e}}\right.$ année, $\left.\mathrm{n}^{\circ} 1161\right)-30$ nov. $1972\left(\mathrm{n}^{\circ} 6127\right)$. Bayonne, $44 \times 30$ puis $59 \times 44 \mathrm{~cm}$

En français. - Numérotation erronée. - Suite de : Résistance (La) républicaine. - Texte en partie identique puis en sept. 1951 identique à celui de: Biarritz-soir. - Fusionne avec: Biarritz-soir; Gazette (La) luzienne; Nouvelle (La) gazette de Biarritz et Soir (Le) de Bayonne pour former : Echo (L') du Sud-Ouest

Côté sorties. Le mensuel gratuit des sorties et spectacles [puis des sorties, spectacles et sports, Pays basque et Sud-Landes]. avr. $2004 \rightarrow$. Anglet, $24 \times 17 \mathrm{~cm}$ En français

137 * Coulée (La) rouge. Bulletin [des] communistes marxistes-léninistes des Fonderies [de Mousseroles]. [févr. 1977] $\left(\mathrm{n}^{\circ} 1\right)$ - [...]. - [Bayonne], $30 \times 21 \mathrm{~cm}$

Titre basque : Kulaia gorria. - En français. - Publ. par : Parti communiste marxiste-léniniste de France. Cellule Robert Barnetche [Bayonne]. - Voir aussi Bulletin de la Cellule Barnetche du P.C.M.L.

138 Coup de pouce. Bulletin de liaison Tec. Ge. Coop. [puis Edité par le groupe Tec. Ge. Coop.]. Irrégulier. [1984/85 - 1989] $\left(12 \mathrm{n}^{\circ}\right)$ [?]. - Bayonne, $41 \times 29$ puis $30 \times 21 \mathrm{~cm}$

En français. - Publ. par : Tec. Ge. Coop. [Technique-Gestion-Coopération] [Bayonne]

139 Courrier (Le). A refusé de paraître sous l'occupation [puis Sabordé le $1^{\text {er }}$ juillet 1940 puis Fondé en 1829...]. Quotidien puis hebdomadaire. 11 sept. $1944-29$ juin 1968 (138 année, ${ }^{\circ}$ 32801). 6 - 27 juil. 1968 (138 année, n'1-4). - Bayonne, Biarritz, $45 \times 30$ ou $60 \times 45$ puis $64 \times 43$ puis $43 \times 32 \mathrm{~cm}$

En français; quelques textes basques, irrégulièrement. - Dir. Jean de l'Espée. - Numérotation erronée. - Suite de: Courrier (Le) de Bayonne [4 avr. 1852 - $1^{\mathrm{er}}$ juil. 1940]. - Le 17 nov. 1947 absorbe : Eclaireur (L') du Sud-Ouest et jusqu'au 30 nov. 1951 parait sous le titre : Courrier (Le), L'Eclaireur. - A partir du 1er août 1959 texte en partie identique à celui de : Républicain (Le) du Sud-Ouest. - Texte identique ou en partie identique à celui de : Journal de Biarritz et de la Côte basque, qu'il absorbe le 22 mars 1965; parait alors sous le titre: Courrier (Le), Journal de Biarritz. - Voir aussi Courrier (Le) agricole

140 Courrier (Le) agricole. Hebdomadaire. 1950 [?] - [...]. - Bayonne, $56 \times 40$ puis $58 \times 43 \mathrm{~cm}$ Paraissait en 1957 et 1958. - En français; quelques textes basques et gascons. - Même adresse et même dir., Jean de l'Espée, que : Courrier (Le)

141 Courrier (Le) de Bayonne. Edition bayonnaise de la Gazette de Biarritz. Hebdomadaire paraissant le vendredi. [17 juin - 2 sept. 1988] (n¹-12) [?]. - Biarritz, $36 \times 26$ puis $34 \times 25$ $\mathrm{cm}$ En français

142 Courrier (Le) de Vasconie. Journal trimestriel. janv. 1986 - mars 1987 (n¹-5). - La Testede-Buch, $32 \times 22 \mathrm{~cm}$

Porte au-dessus du titre: Nouvelle édition en hommage à Augustin Chaho, créateur en 1844, à Bayonne, du journal de même titre. - En français; quelques textes basques, espagnols et gascons. - Publ. par : Association vasconne d'études et d'initiatives [La Teste-de-Buch]

Crack-bulle voir Krack-bulle 
144 * Crayon bleu. [...]. - Bayonne

Publ. par: Rayon bleu, association des élèves du BTS Audiovisuel, au Lycée René Cassin [Bayonne]. - Aurait paru après 1988, année de création de l'association. - En 1997 remplacé par : Mire de Barges

Croisés (Les) de Saint-André. Annuel. [1997/98] $\rightarrow$. Bayonne, 30 × $21 \mathrm{~cm}$

En français. - Publ. par : Croisés (Les) de Saint-André [Bayonne]. - Suite de : Patro (Le)

Croix (La) du Sud. Bulletin de liaison des thoniers africains et des familles maritimes de St-Jean-de-Luz, Ciboure, Socoa. 1956/57 - 1960/61. - Saint-Jean-de-Luz, $27 \times 22 \mathrm{~cm}$

En français; quelques textes basques. - Paraît durant les campagnes de pêche. - Remplacé par : Jeiki jeiki

147 Cuba siempre. Bulletin d'information du Comité Pays basque/Bas-Adour de France-Cuba [puis Association France-Cuba. Bulletin d'information...]. Trimestriel. mars $1998\left(\mathrm{n}^{\circ} 1\right) \rightarrow$. Bayonne, $30 \times 21 \mathrm{~cm}$

Avec un ño de déc. 1997. - Le sous-titre varie. - En français

Cultzine. \{Pays basque-Sud des Landes\} [puis Pays basque-Landes puis Pays basque, Landes, Béarn, Bigorre]. \{Art, culture, cinéma, concert, musique\}. Agenda des sorties... Mensuel. déc. $1999\left(n^{\circ} 1\right) \rightarrow$. Ciboure, $15 \times 11 \mathrm{~cm}$

En français. - En août 2003 absorbe l'éd. Béarn-Bigorre, qui paraissait parallèlement depuis mars 2002; voir la notice suivante

Cultzine. Béarn-Bigorre. Agenda des sorties \{Béarn-Bigorre\}. Mensuel. mars 2002 - juil. 2003 ( $1^{\text {re }}-2^{\mathrm{e}}$ année, $\left.\mathrm{n}^{\circ} 1-16\right)$. - Ciboure, $15 \times 11 \mathrm{~cm}$

En français. - Absorbé par l'éd. Pays basque-Landes; voir la notice précédente

\section{INDEX}

Index chronologique : 20e siècle

Index géographique : Pays basque (France)

Thèmes : bibliographie

Mots-clés : publication périodique 